\title{
Prevalência de problemas de saúde mental na infância na atenção primária
}

\author{
Childhood Mental Health Problems in Primary Care
}

Daniel Fatori ${ }^{1}$

Alexandra Brentani ${ }^{2}$

Sandra Josefina Ferraz Ellero Grisi ${ }^{2}$

Euripedes Constantino Miguel ${ }^{1}$

Ana Soledade Graeff-Martins ${ }^{3}$

${ }^{1}$ Departamento de Psiquiatria, Hospital das Clínicas, Faculdade de

Medicina, Universidade de São Paulo (USP). R. Dr Ovídio Pires de Campos 785, Cerqueira César. 05403 010 São Paulo SP Brasil. daniel.fatori@gmail.com

${ }^{2}$ Departamento de Pediatria, Hospital das

Clínicas, Faculdade de

Medicina, USP. São Paulo

SP Brasil.

${ }^{3}$ Departamento de

Psiquiatria e Medicina Legal, Faculdade de Medicina,

Universidade Federal do Rio

Grande do Sul. Porto Alegre

RS Brasil.

\begin{abstract}
The present population study aimed at identifying the prevalence of mental health problems (MHP) and describing health services use in a sample of children attending Primary Healthcare Units (PHU) in the city of Sao Paulo. Caregivers of 825 6-11 years old children were assessed. MHP were assessed with the Strength and Difficulties Questionnaire and health services use with an adapted version of the Client Receipt Inventory Children`s version. Prevalence of internalizing and externalizing MHP was $30.7 \%$ and $18.3 \%$, respectively. Pediatricians were the most consulted health professional (56.7\%) and psychologists the most consulted mental health professional (7.9\%). Only 3 children were under medication treatment for MHP. The high prevalence of MHP among children in the primary care setting and the low rate of treatment constitute a public health problem. Training programs for health professionals are relevant to help identify and refer MHP cases. Key words Primary care, Public health, Child, Epidemiology, Cross-sectional studies, Brazil.
\end{abstract}

Resumo O presente estudo populacional de corte transversal teve como objetivo verificar a prevalência de problemas de saúde mental (PSM) e descrever o uso de serviços de saúde numa amostra de crianças atendidas em unidades de saúde (UBS) do município de São Paulo. Responsáveis de 825 crianças de 6-11 anos de idade foram entrevistados. PSM das crianças foram avaliados pelo "Strength and Difficulties Questionnaire" e uso de serviços por uma versão adaptada do "Client Service Receipt Inventory Children's version”. A prevalência de PSM internalizantes e externalizantes foi de 30,7\% e 18,3\%, respectivamente. O pediatra foi o profissional de saúde mais consultado (56,7\%), o psicólogo foi o profissional da saúde mental mais consultado (7,9\%). Apenas 3 crianças estavam em tratamento medicamentoso para PSM. A alta prevalência de PSM em crianças atendidas na AP e o baixo número de atendimentos em serviços configuram um importante problema de saúde pública. Programas de capacitação para profissionais são importantes para aprimorar a identificação e o encaminhamento de casos de PSM.

Palavras-chave Atenção Primária à Saúde, Saúde pública, Criança, Epidemiologia, Estudos transversais, Brasil 


\section{Introdução}

O paradigma contemporâneo de saúde tem como peça fundamental e indissociável a saúde mental ${ }^{1}$. O alto grau de limitação funcional e sofrimento causado pelos problemas de saúde mental resultam em grave impacto na qualidade de vida e produtividade. Estudo recente aponta que 22,7\% dos anos vividos com incapacidade pela população mundial atualmente (years of life lost to disability; YLD) são decorrentes de problemas de saúde mental (PSM). A epidemiologia demonstra que os PSM frequentemente se iniciam na infância e adolescência $^{2}$, seguindo trajetória crônica ${ }^{2}$.

No Brasil, estima-se que cerca de 13\% da população de crianças e adolescentes têm algum diagnóstico de $\mathrm{PSM}^{3,4}$, com tendência dos sintomas persistirem de forma crônica. Dados brasileiros preliminares sugerem que entre casos graves e crônicos, apenas 37,5\% tenham recebido algum tratamento num período de cinco anos ${ }^{5}$. Essa alta taxa de prevalência, baixa taxa de tratamento, somada à trajetória crônica, torna a Atenção Primária (AP) do Sistema Único de Saúde (SUS) o âmbito ideal para prevenção, avaliação e tratamento dos PSM na infância e adolescência ${ }^{6}$, já que é nesse contexto que a maioria da população brasileira busca atendimento em saúde ${ }^{7}$.

A AP é um conjunto de iniciativas voltadas para a promoção da saúde, prevenção de agravos, tratamento e reabilitação. No Brasil é estruturada por meio da Estratégia da Saúde da Família (ESF), que tem como instrumento principal as equipes de saúde da família atuando nas Unidades Básicas de Saúde (UBS), configurando a porta de entrada do SUS. As equipes são compostas por um médico generalista, uma enfermeira, dois auxiliares de enfermagem e até seis agentes comunitários de saúde. Os profissionais das equipes de saúde da família têm como função avaliar, intervir e encaminhar para serviços especializados, quando necessário. Atualmente, a ESF cobre $62,5 \%$ da população brasileira ${ }^{8}$.

Apesar da relevância da ESF, há uma escassez de dados epidemiológicos e quantitativos sobre o perfil psicopatológico e o padrão de uso de serviços por crianças e adolescentes usuários dos serviços da AP do SUS. Determinar a prevalência de transtornos mentais na infância na rede de AP, assim como o padrão de uso de serviços desta população, pode auxiliar no planejamento da disponibilidade de recursos humanos e oferta de serviços da AP, atenção secundária e terciária. A compreensão dos tipos de PSM e graus de severidade podem ainda fornecer dados importantes para o planejamento da rede de atenção psicossocial (RAPS) ${ }^{9}$ com o intuito de oferecer uma dinâmica de atendimento e encaminhamentos mais eficiente.

Assim, o presente estudo tem como objetivo: (1) verificar a prevalência de PSM em uma amostra de crianças atendidas em UBSs da rede de AP de uma região do município de São Paulo; (2) descrever o uso de serviços de saúde nessa mesma amostra.

\section{Método}

Estudo populacional de corte transversal realizado em cinco UBSs da região oeste do município de São Paulo. O processo de amostragem se constituiu de um levantamento de todos os pacientes na faixa etária de 6-11 anos de idade que realizaram consultas (com atendimento registrado no Sistema Integrado de Gestão de Atendimento, SIGA) nessas UBSs nos meses de dezembro de 2011 a março de $2012(\mathrm{~N}=1423)$. As cinco UBSs mencionadas fizeram parte do projeto "Promoção da saúde mental na estratégia saúde da família: intervenção precoce visando à prevenção dos transtornos mentais e seu impacto socioeconômico", financiado pela Fundação de Amparo à Pesquisa do Estado de São Paulo (FAPESP), que capacitou profissionais de saúde no que tange à identificação e manejo de problemas de saúde mental na infância e adolescência ${ }^{10}$.

A coleta de dados foi realizada nos primeiros quatro meses de 2014. Os entrevistadores eram profissionais com ensino médio que receberam treinamento por pesquisador com experiência em estudos epidemiológicos. Os entrevistadores foram instruídos a entrarem em contato com as famílias por meio de visitas domiciliares. Nos casos em que as famílias não eram encontradas na primeira tentativa, os entrevistadores fizeram uma busca ativa no bairro/região. Todas as entrevistas aconteceram nos domicílios das famílias. Ao final deste processo foi possível localizar e entrevistar 825 pais/responsáveis das crianças (perda amostral: 42\%).

Os seguintes instrumentos de avaliação foram utilizados:

Versão adaptada do Client Service Receipt Inventory Children's version - questionário desenvolvido por pesquisadores do King's College London para levantar dados acerca do uso de serviços de saúde, tratamentos (incluindo medicamentoso e psicoterápico), serviços sociais e educacionais, entre outros ${ }^{11}$, na infância e ado- 
lescência. A versão brasileira possui nove itens principais. $\mathrm{O}$ tempo de duração de aplicação do instrumento pode variar entre 20 e 40 minutos. A seguir, uma breve descrição acerca dos itens relativos à presente pesquisa. Sobre o uso de serviços de saúde, pergunta-se acerca de: pediatra, clínico geral, médico de família, psicólogo, psicopedagoga, fonoaudióloga, assistente social, terapeuta de família, terapeuta ocupacional, neurologista, psiquiatra, Centro de Atenção Psicossocial (CAPS) e outros. Atendimento por psiquiatra, psicólogo ou em CAPS também foi agregado em uma única variável: "qualquer profissional ou serviço especializado em saúde mental". Ressalta-se que o uso de serviços independe da queixa original. Há um item sobre internação por qualquer razão médica. Por fim, uma pergunta sobre o uso de qualquer medicamento para tratamento de problemas emocionais, de comportamento ou com álcool e/ou drogas com descrição de dose diária, data de início e fim do tratamento. Todas as perguntas se referem aos últimos 12 meses.

Strength and Difficulties Questionnaire (SDQ, Questionário de Capacidades e Dificuldades) questionário que avalia crianças e adolescente na faixa etária de quatro a 16 anos e possui 20 itens distribuídos em cinco escalas: problemas emocionais, problemas de conduta, hiperatividade/déficit de atenção, problemas de relacionamento com colegas e comportamento social positivo, além da soma das primeiras quatro escalas, que representa o total de dificuldades (www.sdqinfo.com). A versão brasileira do SDQ se mostrou adequada para uso em estudo de validaçãa ${ }^{12}$ e tem sido utilizada em diversos estudos brasileiros ${ }^{13}$. Os sujeitos de pesquisa foram categorizados por meio das escalas de problemas internalizantes (itens de problemas emocionais e de relacionamento com colegas) e externalizantes (problemas de conduta e hiperatividade/déficit de atenção), cada uma com escore entre $0-20$. O ponto de corte para a categoria de nível clínico foi de $\geq 8$ pontos para internalizantes $\mathrm{e} \geq 11$ pontos para externalizantes. Essa forma de categorização e respectivos pontos de corte se demonstraram adequados em estudo populacional prévio ${ }^{14}$. Optou-se por estas categorias com o intuito de verificar possíveis diferenças entre as duas dimensões de sintomatologia já consagradas na literatura.

O estudo teve aprovação da Comissão de Ética em Pesquisa da Faculdade de Medicina da Universidade de São Paulo. As entrevistas foram realizadas após o consentimento por escrito das mães/responsáveis, mantendo-se o sigilo das informações.
Foi realizada análise estatística descritiva das variáveis de interesse do estudo. Verificaram-se possíveis associações entre as variáveis por meio do teste de qui-quadrado (teste exato de Fisher) e as odds ratio (OR; razão de chance) foram reportadas para todos os cruzamentos entre variáveis.

\section{Resultados}

A amostra total foi de 825 crianças de 6-11 anos de idade (média: 8,23, desvio-padrão: 1,52), sendo que $49,9 \%$ eram do sexo masculino. A distribuição de crianças da amostra quanto à cor de pele foi: branca $(57,6 \%)$, negra $(10,6 \%)$, entre branca e negra $(31,5 \%)$ e indígena $(0,2 \%)$. A prevalência de PSM internalizantes (PSMI) e externalizantes (PSME) foi de $30,7 \%$ e $18,3 \%$, respectivamente (ambos em nível clínico de acordo com o SDQ).

O pediatra foi o profissional de saúde mais consultado nos 12 meses antecedentes à entrevista com a mãe/responsável (56,7\%), seguido do médico de família $(47,6 \%)$ e clínico geral $(19,0 \%)$. Dentre os profissionais de saúde mental, o psicólogo foi o mais consultado (7,9\%), sendo que apenas $1,1 \%$ das crianças passaram por consulta com psiquiatra nos últimos 12 meses. Somente três crianças estavam em atendimento no CAPS. Nenhuma das 27 internações hospitalares nos últimos 12 meses aconteceram em razão de problema de saúde mental (Tabela 1).

Apenas três crianças $(0,4 \%)$ estavam em tratamento medicamentoso para problemas de saúde mental nos últimos 12 meses. Os medicamentos psiquiátricos utilizados eram: valproato de sódio (sujeito 1), carbamazepina, fenorbital e diazepam (sujeito 2), cloridrato de clomipramina (sujeito 3).

Dentre crianças com PSM, o pediatra foi o profissional de saúde mais consultado nos últimos 12 meses $(61,1 \%$ e $56,4 \%$, entre crianças com PSMI e PSME, respectivamente). No mesmo período de tempo 53,2\% (PSMI) e 33,8\% (PSME) destas crianças passaram por consulta com algum profissional ou em serviço especializado em saúde mental (psicólogo, terapeuta ocupacional, terapeuta familiar, psiquiatra ou CAPS) (Tabela 2).

Atendimentos nas seguintes especialidades nos últimos 12 meses demonstraram associação estatística com PSM (Tabela 2): médico de família (PSMI: OR 1,4, p < 0,05), psicólogo (PSMI: OR 2,4, p < 0,001, PSME: OR 2,7, p < 0,001), psicopedagogo (PSMI: OR 4,1, $\mathrm{p}<0,05$ ), fono- 
audiólogo (PSMI: OR 1,8, p <0.05), neurologista (PSMI: OR 4,2, p $<0,001$ ), terapeuta ocupacional (PSME: OR 4,5, p < 0,05). Ter recebido atendimento por qualquer profissional ou em serviço especializado em saúde mental também se mostrou associado estatisticamente (PSMI: OR 2,8, p $<0,001$, PSME: OR 2,5, $\mathrm{p}<0,001)$.

Tabela 1. Uso de serviços de saúde por crianças de 6-11 anos de idade nos últimos 12 meses $(\mathrm{N}=825)$.

\begin{tabular}{lr}
\hline & \multicolumn{1}{c}{$\mathbf{N}(\%)$} \\
\hline Pediatra & $455(56,7 \%)$ \\
Ménico Geral & $149(19,0 \%)$ \\
Neurologista & $383(47,6 \%)$ \\
Psiquiatra & $25(3,1 \%)$ \\
Psicólogo & $9(1,1 \%)$ \\
Psicopedagogo & $63(7,9 \%)$ \\
Fonoaudiólogo & $22(2,8 \%)$ \\
Assistente Social & $59(7,4 \%)$ \\
Terapeuta de família & $45(5,6 \%)$ \\
Terapeuta ocupacional & $1(0,1 \%)$ \\
CAPS & $8(1,0 \%)$ \\
Outros & $3(0,4 \%)$ \\
Internação hospitalar & $71(8,6 \%)$ \\
Qualquer serviço de saúde geral & \\
Qualquer serviço de saúde mental $^{\mathrm{b}}$ & $639(78,7 \%)$ \\
\hline a atendimento por pediatra, clínico geral ou médico de \\
família. ${ }^{\mathrm{b}}$ atendimento por psiquiatra, psicólogo ou em
\end{tabular}

\section{Discussão}

O presente estudo investigou a prevalência de PSM em crianças atendidas em UBSs da rede de atenção primária do município de São Paulo. A prevalência de PSM internalizantes e externalizantes foi de $30,7 \%$ e $18,3 \%$, respectivamente. As altas taxas encontradas se assemelham a alguns estudos conduzidos em amostras comunitárias brasileiras. Por exemplo, um estudo em Ribeirão Preto $(\mathrm{N}=107)$ avaliou crianças de 6-11 anos de idade com o SDQ e encontrou as seguintes taxas: $30,8 \%$ de problemas emocionais, $14 \%$ de problemas interpessoais, $17,7 \%$ de problemas de conduta, $16,8 \%$ de hiperatividade ${ }^{15}$. Em Taubaté, também com o uso do SDQ em amostra comunitária, foi encontrada a taxa de $22,7 \%$ de total de problemas (nível clínico) em crianças de 7-11 anos ( $\mathrm{N}=$ $454)^{16}$. Em Pelotas, a taxa de problemas de atenção e hiperatividade (único desfecho avaliado) foi de 19,9\% em crianças de 11 anos de idade $(\mathrm{N}=$ 4.423). Outros estudos brasileiros, também com o uso do SDQ, encontraram prevalências menores do que o presente estudo. Em um estudo conduzido na Ilha da Maré, Bahia $(\mathrm{N}=519)$, pesquisadores encontraram uma taxa de total de problemas de 9,8\% usando informações de múltiplos informantes em crianças de 7-14 anos ${ }^{17}$. Já numa amostra de 327 crianças e adolescentes de 11-15 anos de idade no município de Campos do Jordão, pesquisadores encontraram uma taxa de total de problemas de $12,5 \%{ }^{18}$. Ressalta-se que dado o fato da presente amostra advir de unidades de saúde era esperado que a prevalência encontrada

Tabela 2. Uso de serviços de saúde por crianças de 6-11 anos de idade nos últimos 12 meses por tipo de problema de saúde mental segundo o SDQ $(\mathrm{N}=825)$.

\begin{tabular}{lrrrr}
\hline & \multicolumn{2}{c}{ Problemas Internalizantes } & \multicolumn{2}{c}{ Problemas Externalizantes } \\
\cline { 2 - 5 } & \multicolumn{1}{c}{ Sim } & OR (IC 95\%) & \multicolumn{1}{c}{ Sim } & OR (IC 95\%) \\
\hline Pediatra & $151(61,1 \%)$ & $1,3(0,9-1,8)$ & $84(56,4 \%)$ & $1,0(0,7-1,4)$ \\
Clínico Geral & $54(22,2 \%)$ & $1,3(0,9-2,0)$ & $31(21,1 \%)$ & $1,2(0,8-1,8)$ \\
Médico Família & $131(53,3 \%)$ & $1,4(1,0-1,9)^{*}$ & $77(52,4 \%)$ & $1,3(0,9-1,8)$ \\
Psicólogo & $33(13,3 \%)$ & $2,7(1,6-4,6)^{* *}$ & $22(14,9 \%)$ & $2,7(1,5-4,6)^{\star *}$ \\
Psicopedagogo & $14(5,7 \%)$ & $4,1(1,7-9,9)^{*}$ & $6(4,2 \%)$ & $1,7(0,7-4,5)$ \\
Fonoaudiólogo & $25(10,2 \%)$ & $1,8(1,0-3,1)^{*}$ & $8(5,5 \%)$ & $0,7(0,3-1,5)$ \\
Assistente Social & $20(8,2 \%)$ & $1,9(1,0-3,4)$ & $14(9,6 \%)$ & $2,1(1,1-4,1)$ \\
Terapeuta familiar & 0 & $0,7(0,7-0,7)$ & & $0,9(0,8-0,8)$ \\
Neurologista & $16(6,5 \%)$ & $4,2(1,8-9,6)^{* *}$ & $8(5,4 \%)$ & $2,1(0,9-5,1)$ \\
Terapeuta ocupacional & $4(1,6 \%)$ & $2,3(0,6-9,1)$ & $4(2,7 \%)$ & $4,5(1,1-18,3)^{*}$ \\
Psiquiatra & $5(2 \%)$ & $2,9(0,8-10,7)$ & $4(2,8 \%)$ & $3,7(1,0-13,8)$ \\
CAPS & $2(0,8 \%)$ & $4,5(0,4-50,2)$ & $2(1,4 \%)$ & $9,1(0,8-100,6)$ \\
\hline$*$
\end{tabular}

${ }^{*} \mathrm{p}<0,05 .{ }^{* *} \mathrm{p} \leq 0,001$. 
fosse maior do que em amostras comunitárias de faixa etária semelhante.

Até a década de 1980 a taxa de PSM em crianças e adolescentes na atenção primária era estimada em $4-15 \%{ }^{19}$. Em 1988, um estudo nos Estados Unidos com 789 crianças de 7-11 anos encontrou uma taxa de 24,7\% usando o Child Behavior Checklist $(\mathrm{CBCL})^{20}$. Nas décadas seguintes foram conduzidos inúmeros estudos sobre o tema.

Um estudo realizado nos Estados Unidos avaliou 21.065 crianças e adolescente de 4-15 anos, em toda a rede nacional de AP, e encontrou $13 \%$ de PSM segundo o Pediatric Symptom Checklist ${ }^{21}$. Em outro estudo norte-americano, com amostra de 767 crianças de 5-16 anos, 28\% apresentaram alta sintomatologia de acordo com o SDQ ${ }^{22}$. Os dois estudos encontraram taxas de PSM inferiores aos dados da presente amostra.

Alguns estudos avaliaram especificamente PSMI em amostras de pacientes da AP. Um estudo nos Estados Unidos avaliou 714 crianças e adolescentes de 8-17 anos e encontrou 20\% de sintomas de ansiedade segundo o instrumento Screen for Anxiety Related Emotional Disorders ${ }^{23}$. Na França, pesquisadores avaliaram 155 crianças e adolescentes (7-17 anos de idade) que receberam atendimento na AP. Entre indivíduos com menos de 13 anos foi encontrada uma prevalência de depressão de 10\% segundo o instrumento Center for Epidemiological Studies Depression ${ }^{24}$. Estas duas pesquisas encontraram taxas significativamente menores do que a de 30,7\% de PSMI do presente estudo, apesar de também terem utilizado instrumentos de rastreamento.

Uma revisão sistemática verificou a prevalência de transtorno de déficit de atenção e hiperatividade (TDAH) na infância/adolescência em pacientes da AP e encontrou a taxa de $11,2 \%$ na faixa etária de 6-12 anos de idade ${ }^{25}$. Um grande estudo epidemiológico avaliou 22.059 crianças e adolescentes com 4-15 anos de idade nos Estados Unidos e Canadá, encontrando uma prevalência de TDAH de 9,2\% segundo avaliação clínica ${ }^{26}$. A prevalência de PSME encontrada na amostra do presente estudo foi maior do que em ambos. Contudo, estes se utilizaram de diagnósticos segundo critérios do Diagnostic and Statistical Manual of Mental Disorders (DSM), tornando a avaliação mais específica e menos inclusiva. Além disso, PSME segundo o SDQ incluem problemas de conduta e hiperatividade, tornando a categoria mais ampla.

No Brasil apenas um único estudo avaliou PSM em amostra de serviços da rede de atenção primária. Avaliando com o SDQ crianças de 5-11 anos que passaram por atendimento pediátrico em UBS, foi encontrado 55\% de casos de PSM $^{27}$, uma taxa muito mais alta que a encontrada no presente estudo. Contudo, os autores não descreveram como classificaram os casos de PSM, sendo possível terem incluído casos limítrofes na prevalência.

Os PSMI apresentaram associações com ter realizado atendimento nos últimos 12 meses com médico de família, psicólogo, psicopedagogo, fonoaudiólogo e neurologista, enquanto os PSME apresentaram associação com ter sido atendido por terapeuta ocupacional e psicólogo. A literatura internacional mostra que PSME costumam receber mais atenção em saúde do que $\mathrm{PSMI}^{28}$. Contudo, um estudo epidemiológico brasileiro que avaliou 1.721 crianças de 6 a 16 anos de idade de quatro regiões do país demonstrou que o uso de serviços de saúde mental nos últimos 12 meses estava associado aos transtornos mentais disruptivos e de ansiedade ${ }^{29}$. Isto é, tanto problemas do tipo internalizantes, como externalizantes, estavam associados ao uso de serviços de saúde mental. Possivelmente, a população e os serviços de saúde mental brasileiros seguem uma tendência diferente, dessa forma, novos estudos serão necessários para compreender este tópico.

Outra possibilidade é que os PSMI apresentem-se por meio de sintomas somáticos (como ocorre nos sintomas de ansiedade e depressão), levando os pais a procurarem ajuda. Dessa forma, sendo a AP a porta de entrada do SUS, poderia haver uma tendência dos pais levarem os filhos com sintomas somáticos à AP. Considerando que professores podem apresentar boa capacidade de identificação de PSME em crianças após capacitação ${ }^{30}$, sugere-se uma aproximação da escola com a rede de AP para uma melhor identificação de casos que necessitam de tratamento. Neste sentido, já existem programas efetivos disponíveis para professores escolares reconhecerem transtornos mentais na infância ${ }^{31}$. Por fim, uma hipótese alternativa para compreender as associações estatísticas de atendimentos com PSME se dá possivelmente pelo viés devido a perda amostral.

De forma geral, o perfil de uso de serviço na amostra apresentou pouco uso de atendimento psicológico $(7,9 \%)$ e psiquiátrico $(1,1 \%)$. Entre as crianças com PSM, 53,2\% com PSMI e 33,8\% com PSME receberam algum tratamento especializado em saúde mental. Levando em conta a proposta da AP de avaliar e encaminhar casos que necessitam de atendimento especializado, ressal- 
ta-se o baixo número de crianças com PSM recebendo atendimento adequado, principalmente aquelas com PSME. Isso pode se dar em razão da baixa capacidade dos profissionais da AP em avaliar adequadamente casos que precisam de atenção especializada, ou à falta de profissionais especializados na $\mathrm{AP}$, ou ainda à dificuldade dos pais em perceber os sintomas de PSM como algo que necessita de tratamento. Também é possível que isso ocorra em função da carência de serviços especializados em saúde mental disponíveis, uma vez que a escassez de profissionais especialistas em saúde mental de crianças e adolescentes se verifica em todo mundo. Estudos brasileiros recentes, em diversas regiões do país, já demonstraram que entre 20\%-37\% das crianças e adolescentes com PSM recebem algum atendimento ${ }^{5,29}$.

$\mathrm{O}$ pediatra foi o profissional mais acessado na amostra $(56,7 \%)$, seguido do médico da família (47,6\%). Este dado já era esperado, justamente devido ao fato destes profissionais serem os mais disponíveis na rede de AP. Assim, pode ser interessante implantar programas de capacitação para que estes profissionais possam identificar e tratar os PSM mais comuns. Estudos têm demonstrado que programas de capacitação em saúde mental da infância para pediatras e para o médico dos programas de saúde da família são eficazes e trazem benefícios como mudança de atitude e melhor capacidade de encaminhamento $^{10}$. Como, entre os profissionais de saúde mental, o psicólogo se mostrou o mais procurado ${ }^{29}$, especial atenção deve ser dada na formação destes profissionais para que estes possam realizar diagnósticos e intervenções baseadas em evidências e adequadas a esta faixa etária.

Nos últimos anos o tema do uso de medicamentos psiquiátricos na infância tem surgido na mídia $^{32,33}$ e em artigos científicos ${ }^{34}$. Contudo, as evidências apresentadas usualmente são inadequadas ou anedóticas, além de raramente pautadas em dados epidemiológicos. Numa amostra de 825 crianças atendidas na rede de AP foram encontradas apenas três crianças que fizeram uso de medicamentos psiquiátricos no último ano (e ainda assim, em pelo menos dois dos três casos, é possível que a criança estivesse usando medicamentos para o tratamento de doença neurológica), contrariando relatos que apontam prescrição excessiva de medicamentos psiquiátricos na infância. Assim, pelo contrário, a baixa prescrição de medicamentos psiquiátricos aliada a alta prevalência de PSM relatada neste artigo, reflete, possivelmente, a desassistência e a falta de cuidados adequados a esta população. Um estudo prévio demonstrou que o transtorno de déficit de atenção e hiperatividade é subtratado no Brasil ${ }^{35}$, corroborando com esta hipótese. Isto, aliado a evidências que demonstram que mais de $60 \%$ das crianças e adolescentes com transtornos mentais não recebem atendimento especializado em saúde mental ${ }^{5,29}$, revelam um cenário geral de desassistência. Além disso, o Brasil possui apenas 3,5 psiquiatras para cada 100 mil habitantes. Países de alta renda como Estados Unidos e Reino Unido têm 12,4 e 14,6 psiquiatras por 100 mil habitantes, respectivamente ${ }^{36}$. Logo, a baixa frequência de medicação na presente amostra pode ser explicada pelo baixo número de psiquiatras no Brasil.

O presente estudo tem limitações. A perda amostral de $42 \%$ caracteriza um importante viés de seleção, uma vez que é impossível saber o que ocorreu com grande parte da amostra selecionada. Uma parcela dessa perda se dá ao fato de muitas famílias terem se mudado da região original de atendimento. Além disso, não foi possível comparar os sujeitos que não foram encontrados com a amostra final, pois as listas de pacientes obtidas não apresentavam informações sociodemográficas. Como a pesquisa se delimitou a uma região específica de apenas um município, não é possível generalizar os dados para demais regiões do município, muito menos para outras cidades e regiões do país. Por último, as avaliações dos sintomas de PSM foram conduzidas por meio de um instrumento de rastreamento (SDQ), não sendo possível determinar os critérios diagnósticos (segundo DSM) específicos de cada caso avaliado, nem definir se o caso é realmente positivo.

A alta prevalência de PSM em crianças atendidas na rede de AP se comparada a estudos internacionais e o baixo número de atendimentos em serviços especializados configuram um problema importante, principalmente se levarmos em conta que as UBSs estudadas encontram-se em região central da maior cidade do Brasil. Programas de capacitação para profissionais da AP, particularmente o pediatra e o médico da família, além de especial atenção a melhor qualificação do psicólogo, são importantes para melhorar a identificação de PSM na infância e, assim, aprimorar os encaminhamentos para serviços especializados. Quanto mais cedo os PSM na infância forem identificados e adequadamente tratados, melhor o curso e o prognóstico. Dessa forma, todo investimento em programas baseados em evidências focados nesta faixa etária configuram investimento da mais suma importância. 


\section{Colaboradores}

D Fatori, A Brentani, SJFE Grisi e EC Miguel contribuíram igualmente na elaboração do manuscrito.

\section{Referências}

1. Prince M, Patel V, Saxena S, Maj M, Maselko J, Phillips MR, Rahman A. No health without mental health. Lancet 2007; 370(9590):859-877.

2. Kim-Cohen J, Caspi A, Moffitt TE, Harrington H, Milne BJ, Poulton R. Prior juvenile diagnoses in adults with mental disorder: developmental follow-back of a prospective-longitudinal cohort. Arch Gen Psychiatry 2003; 60(7):709-717.

3. Paula CS, Coutinho ES, Mari JJ, Rohde LA, Miguel EC, Bordin IA. Prevalence of psychiatric disorders among children and adolescents from four Brazilian regions. Rev Bras Psiquiatr 2015; 37(2):178-179.

4. Fleitlich-Bilyk B, Goodman R. Prevalence of child and adolescent psychiatric disorders in southeast Brazil. $J$ Am Acad Child Adolesc Psychiatry 2004; 43(6):727-734.

5. Fatori D, Evans-Lacko S, de Paula C. Child mental health care in Brazil: barriers and achievements. Lancet 2012; 379(9812):e16-e17.

6. Lauridsen-Ribeiro E, Tanaka O. Organização de serviços no Sistema Único de Saúde para o cuidado de crianças e adolescentes com problemas de saúde mental. In: Lauridsen-ribeiro E, Tanaka OY, organizadores. Atenção em Saúde Mental para Crianças e Adolescentes no SUS. São Paulo: Hucitec; 2017. p. 147-169.

7. Paim J, Travassos C, Almeida C, Bahia L, Macinko J. The Brazilian health system: history, advances, and challenges. Lancet 2011; 377(9779):1778-1797.

8. Brasil. Ministério da Saúde (MS). Departamento de Atenção Básica. Histórico de Cobertura da Saúde da Família [Internet]. [acessado 2015 Mar 27]. Available from: http://dab.saude.gov.br/portaldab/dab.php

9. Brasil. Ministério da Saúde (MS). Portaria n ${ }^{\circ} 3.088$, de 23 de dezembro de 2011. Institui a Rede de Atenção Psicossocial para pessoas com sofrimento ou transtorno mental e com necessidades decorrentes do uso de crack, álcool e outras drogas, no âmbito do Sistema Único de Saúde (SUS). Diário Oficial da União 2011; 24 dez.

10. Lowenthal R. Capacitação em saúde mental na infância e adolescência para profissionais da atenção primária [Internet]. [acessado 2015 Abr 16]. Universidade Presbiteriana Mackenzie; 2012. Disponível em: http:// up.mackenzie.br/stricto-sensu/disturbios-do-desenvolvimento/teses-e-dissertacoes-detalhada/artigo/capacitacao-em-saude-mental-na-infancia-e-adolescencia-para-profissionais-da-atencao-primari/

11. Beecham J, Knapp M. Costing psychiatric interventions. In: Thornicroft G, Brewin C, Wing J, editors. Measuring Mental Health Needs. London: Gaskell; 2001. p. 200-224.

12. Woerner W, Fleitlich-Bilyk B, Martinussen R, Fletcher J, Cucchiaro G, Dalgalarrondo P, Lui M, Tannock R. The Strengths and Difficulties Questionnaire overseas: evaluations and applications of the SDQ beyond Europe. Eur Child Adolesc Psychiatry 2004; 13(Supl. 2):II47-54.

13. Fleitlich B, Goodman R. Social factors associated with child mental health problems in Brazil: cross sectional survey. BMJ 2001; 323(7313):599-600. 
14. Goodman A, Lamping DL, Ploubidis GB. When to Use Broader Internalising and Externalising Subscales Instead of the Hypothesised Five Subscales on the Strengths and Difficulties Questionnaire (SDQ): Data from British Parents, Teachers and Children. J Abnorm Child Psychol 2010; 38(8):1179-1191.

15. Cury CR, Golfeto JH. Strengths and difficulties questionnaire (SDQ): a study of school children in Ribeirão Preto. Rev Bras Psiquiatr 2003; 25(3):139-145.

16. Vitolo YLC, Fleitlich-Bilyk B, Goodman R, Bordin IAS. Parental beliefs and child-rearing attitudes and mental health problems among schoolchildren. Rev Saude Publica 2005; 39(5):716-724.

17. Goodman R, Neves dos Santos D, Robatto Nunes AP, Pereira de Miranda D, Fleitlich-Bilyk B, Almeida Filho N. The Ilha de Maré study: a survey of child mental health problems in a predominantly African-Brazilian rural community. Soc Psychiatry Psychiatr Epidemiol 2005; 40(1):11-17.

18. Paula CS, Vedovato MS, Bordin IAS, Barros MGSM, D'Antino MEF, Mercadante MT. Mental health and violence among sixth grade students from a city in the state of São Paulo. Rev Saude Publica 2008; 42(3):524528.

19. Costello EJ. Primary Care Pediatrics and Child Psychopathology: A Review of Diagnostic, Treatment, and Referral Practices. Pediatrics 1986; 78(6):1044-1051.

20. Costello E, Costello A, Edelbrock C, Burns B, Dulcan M, Brent D, Janiszewski S. Psychiatric disorders in pediatric primary care: Prevalence and risk factors. Arch Gen Psychiatry 1988; 45(12):1107-1116.

21. Jellinek MS, Murphy JM, Little M, Pagano ME, Comer DM, Kelleher KJ. Use of the Pediatric Symptom Checklist to screen for psychosocial problems in pediatric primary care: a national feasibility study. Arch Pediatr Adolesc Med 1999; 153(3):254-260.

22. Brown JD, Wissow LS. Screening to identify mental health problems in pediatric primary care: Considerations for practice. Int J Psychiatry Med 2010; 40(1):119.

23. Chavira DA, Stein MB, Bailey K, Stein MT. Child anxiety in primary care: Prevalent but untreated. Depress Anxiety 2004; 20(4):155-164.

24. Mathet F, Martin-Guehl C, Maurice-Tison S, Bouvard M-P. Prevalence of depressive disorders in children and adolescents attending primary care. A survey with the Aquitaine Sentinelle Network. L'Encéphale 2003; 29(5):391-400.

25. Brown RT, Freeman WS, Perrin JM, Stein MT, Amler RW, Feldman HM, Pierce K, Wolraich ML. Prevalence and assessment of attention-deficit/hyperactivity disorder in primary care settings. Pediatrics 2001; 107(3):E43.

26. Wasserman RC, Kelleher KJ, Bocian A, Baker A, Childs GE, Indacochea F, Stulp C, Gardner WP. Identification of attentional and hyperactivity problems in primary care: a report from pediatric research in office settings and the ambulatory sentinel practice network. Pediatrics 1999; 103(3):E38.
27. Tanaka OY, Lauridsen-Ribeiro E. A challenge for primary health care: mental health care implementation. Cad Saude Publica 2006; 22(9):1845-1853.

28. Costello EJ, Copeland W, Cowell A, Keeler G. Service costs of caring for adolescents with mental illness in a rural community, 1993-2000. Am J Psychiatry 2007; 164(1):36-42.

29. Paula CS, Bordin IAS, Mari JJ, Velasque L, Rohde LA, Coutinho ESF. The Mental Health Care Gap among Children and Adolescents: Data from an Epidemiological Survey from Four Brazilian Regions. PLoS ONE 2014; 9(2):e88241.

30. Vieira MA, Gadelha AA, Moriyama TS, Bressan RA, Bordin IA. Evaluating the effectiveness of a training program that builds teachers' capability to identify and appropriately refer middle and high school students with mental health problems in Brazil: an exploratory study. BMC Public Health 2014; 14:210.

31. Pereira CA, Wen CL, Miguel EC, Polanczyk GV. A randomised controlled trial of a web-based educational program in child mental health for schoolteachers. Eur Child Adolesc Psychiatry 2015; 24(8):931-40.

32. Arraes J. Medicalização da infância: o TDAH e suas controvérsias [Internet]. Revista Fórum Semanal. [acessado 2015 Abr 16]. Disponível em: http://revistaforum. com.br/digital/171/medicalizacao-da-infancia-o-tdah -e-suas-controversias/

33. Brum E. O Doping das Crianças. Revista Época [Internet]. 25/02/2013. [acessado 2015 Mar 3]. Disponível em: http://revistaepoca.globo.com/Sociedade/eliane -brum/noticia/2013/02/o-doping-das-criancas.html

34. Decotelli KM, Bohre LCT, Bicalho PPG. The drugs of obedience: medicalization, childhood and biopower: notes on clinic and bolitics. Psicol Ciênc Prof 2013; 33(2):446-459.

35. Mattos P, Rohde LA, Polanczyk GV. ADHD is undertreated in Brazil. Rev Bras Psiquiatr 2012; 34(4):513514.

36. World Health Organization (WHO). WHO Mental Health Atlas 2014 [Internet]. Geneva: WHO. [acessado 2016 Set 11]. Disponível em: http://www.who.int/mental_health/evidence/atlas/mental_health_atlas_2014/ en/

Artigo apresentado em 05/07/2016

Aprovado em 22/09/2016

Versão final apresentada em 24/09/2016 